\title{
Early Detection and Treatment of Subclinical Lymphedema in Patients with Breast Cancer
}

\author{
Aslı Gençay Can, MD, Emel Ekşioğlu, MD, and Fatma Aytül Çakçı, MD
}

\begin{abstract}
Background: Early identification and treatment of subclinical lymphedema may prevent development of advanced stages and its related complications. We aimed to detect subclinical lymphedema and to evaluate the effects of early treatment program on the development of clinical lymphedema, upper extremity functions, and quality of life in patients with subclinical lymphedema.

Methods and Results: Twenty-five women who were diagnosed having subclinical lymphedema were enrolled in the study. The patients were informed about lymphedema and its risk factors, and skin care and exercises to prevent the development of lymphedema. Self-reported symptoms, arm volumes, upper extremity functions, quality of life were evaluated before and after treatment. Tightness and numbness were found to have a good correlation with affected arm volume. Volume of the affected arm, percentage volume difference between the arms, upper extremity functions, and quality-of-life scores improved significantly at the end of the treatment. Conclusions: It is crucial to have early detection and treatment of subclinical lymphedema to prevent the development of established lymphedema in breast cancer survivors. Information about lymphedema and its risk factors, skin care, and home-based specific exercise program improves self-reported symptoms, volume measurements, functioning, and quality of life in patients with subclinical lymphedema.
\end{abstract}

Keywords: subclinical lymphedema, breast cancer, early detection, early treatment

\section{Introduction}

$\mathbf{L}$ YMPHEDEMA IS ONE of the most debilitating complica$\triangle$ tions of breast cancer treatment. ${ }^{1}$ It contributes to impairments in extremity range of motion, muscle strength, arm functioning, activity of daily living, and also psychosocial problems such as anxiety and depression., ${ }^{2,3}$ Once lymphedema manifests itself, it is considered to be a chronic and lifelong disease. ${ }^{1}$ There is no curative treatment for clinically apparent lymphedema. ${ }^{4}$

Clinically apparent lymphedema treatment requires lifelong treatment to control the condition and to prevent complications. ${ }^{4}$ Patients with lymphedema need a lifelong hands-on treatment. Early identification of symptoms, maintaining normal body weight, and early treatment in subclinical stage may prevent development of advanced stages and the related complications. ${ }^{3}$ Clinical lymphedema presents as visible or palpable swelling and may be easily recognized using circumferential limb girth and volumetric measurements. ${ }^{5}$ Volume difference between limbs of $>10 \%$ is generally accepted as clinical lymphedema. ${ }^{6}$ However, there is no generally accepted diagnostic criteria for subclinical lymphedema. The Oncology Section of American Physical Therapy Association and the Dutch Institute for Healthcare Improvement recommend to identify self-reported signs of swelling, heaviness, and numbness during the history, to use bioimpedance analysis, and to assess volume measurements to encourage early detection and diagnosis of subclinical lymphedema.,7

Bioimpedance analysis is recently recommended to be used in subclinical/early-stage lymphedema. However, its measurement is affected by body temperature and body hydration status. ${ }^{7,8}$ The equipment is highly expensive and its high cost limits its usefulness in routine practice. A more economical alternative of bioimpedance analysis holds great promise as an aid in the early detection of subclinical lymphedema. ${ }^{8}$ Because of these, we described our own diagnostic criteria for subclinical lymphedema using self-reported symptoms and volumetric measurements.

There is no evidence-based treatment for subclinical lymphedema. In a few studies in the literature, information

Department of Physical Medicine and Rehabilitation, Yıldırım Beyazıt Dışkapı Research and Training Hospital, University of Health Science, Ankara, Turkey. 
about potential risks of lymphedema, skin care, exercise programs, and compression garments has been suggested for subclinical lymphedema treatment. ${ }^{5,9,10}$ To our knowledge, there is no study to evaluate the effects of treatment on upper extremity function, quality of life, and psychiatric morbidy in subclinical lymphedema patients. We described our own treatment program that included education about risk factors, skin care, and home-based exercise programs and evaluated the effects of this program on the development of clinical lymphedema, upper extremity functions, quality of life, and anxiety and depression in patients with subclinical lymphedema.

\section{Materials and Methods}

One hundred thirty-two women with breast cancer-related surgery, admitted to an outpatient lymphedema clinic with a complaint of arm swelling between September 2017 and April 2018, were assessed for eligibility. The inclusion criteria were as follows: (1) having subclinical lymphedema less than $10 \%$ volume difference between the affected and unaffected arm and the presence of at least one of four selfreported symptoms (swelling, heaviness, tightness, and numbness), (2) unilateral beast cancer, and (3) age between 18 and 65 years. Patients with bilateral breast cancer, a history of trauma or surgery of the affected arm, trunk edema, advanced heart failure, renal insufficiency, and advanced liver disease were excluded from the study. Twenty-five women who met these criteria were enrolled in the study.

This study was performed with the approval of the local ethics committee. All patients provided written informed consent to participate.

\section{Measures}

Medical history and physical examination. Demographic and clinical characteristics of the patients were recorded. History of trauma to the affected arm, response to elevation of the affected arm, and family history, including arm swelling, were evaluated. Patients were asked about the presence of upper extremity symptoms such as numbness, heaviness, tightness, and swelling (self-reported symptoms). In the physical examination, the presence of cellulitis and Stemmer's sign was assessed.

Arm volumes. Affected and unaffected arm volumes were calculated using arm circumference measurements in a formula for frustum volume (truncated cone). ${ }^{11}$ The percentage arm volume difference between the affected and unaffected arm was also calculated. Circumference measurements were taken at four levels-metacarpal, wrist, $10 \mathrm{~cm}$ below the lateral epicondyle, and $10 \mathrm{~cm}$ above the lateral epicondyle with a flexible tape in sitting position with the shoulder $90^{\circ}$ flexion, elbow extended, and forearm pronated. Measurements were taken by the same clinician and recorded in centimeters.

Upper extremity functioning. Upper extremity functions were evaluated using the Quick Disabilities of Arm, Shoulder, and Hand (Q-DASH) questionnaire. It has 11 items and each item is scored from 1 to 5 . Higher scores indicate lower functional level. The validity and reliability of the
Turkish version of the Q-DASH has been done by Düger et al. ${ }^{12}$

Anxiety and depression. The Hospital Anxiety and Depression Scale (HADS) is a commonly used tool for assessing anxiety and depression of the patients with cancer. ${ }^{13}$ It consists of 14 items with anxiety and depression subscales. Each item is scored 0-3. Each subscale is scored between 0 and 21 . Higher scores indicate greater levels of anxiety and depression. Aydemir et al. showed validity and reliability of the Turkish version of the scale. ${ }^{14}$

Quality of life. The quality of life was measured using the SF-36. It is a 36-item questionnaire with 8 domains measuring physical and mental health status (physical component summary [PCS] and mental component summary [MCS], respectively). Each item is scored and summed according to a standardized scoring protocol and each domain is scored between 0 and 100. Higher scores indicate better health status. The validity and reliability of the Turkish version of the SF-36 in cancer patients has been done by Pinar. ${ }^{15}$

\section{Diagnosis of subclinical lymphedema}

Volume difference between limbs of $>10 \%$ is usually accepted as clinically apparent lymphedema, but values $<10 \%$ cannot be used to rule out lymphedema. ${ }^{6}$ Thus, less than $10 \%$ volume difference between the affected and unaffected arm and the presence of at least one of four self-reported symptoms (swelling, heaviness, tightness, and numbness) were accepted as having the diagnosis of subclinical lymphedema.

\section{Interventions}

The patients were informed about lymphedema and its risk factors, skin care, and exercises to prevent the development of lymphedema. They have been given written constent forms and verbal explanations. This treatment program lasts for 1 month.

Lymphedema risk factors. It is important to identify risk factors for the development of lymphedema. All patients were recommended to avoid accidental or inadvertent blood draws/needle sticks, intravenous lines, insect bites, cuts, trauma or blood pressure monitoring in the affected limb, to wear loose jewellery and clothes with no constricting bands, to elevate the limb above the level of the heart, to maintain a normal body weight to prevent both lymphedema and breast cancer recurrence, to avoid exposure to extreme heat and cold, to avoid vigorous activities and to do carefully progressive physical activity with monitoring for symptoms, to drink plenty of water during hot weather, to be alert for the signs of infection (fever, swelling, and redness), and to wear compression garments on flights. ${ }^{2,4,16}$

\section{Skin care}

Because skin hygiene and nail care are important to reduce the risk of infection and cellulitis, all patients were recommended to avoid dry and cracked skin, to use moisturizer creams and soaps with neutral $\mathrm{pH}$, to cut nails straight, to use topical antibiotics to treat small breaks in the skin, to wear gloves while doing activities that may cause skin irritation or 
injury, to wear oven gloves, to use thimble for sewing, to use insect repellent, and to use sunscreen (minimum of SPF 30). ${ }^{16}$

Home-based exercise program. Patients were given a home-based exercise program that included lymphedema remedial exercises, flexibility exercises, stretching exercises, and strengthening exercises. Education about the exercise program was given before initiation of the program. Exercises were recommended three times a day with 10 repetitions. Patients were taught to perform these exercises slowly and sequentially, beginning with the first exercise. The homebased exercise program is shown in Table 1.

\section{Follow-up}

Self-reported symptoms, affected and unaffected arm volumes, upper extremity functions, quality of life, and anxiety/depression conditions of the patients were evaluated before and at the end of treatment (1 month later).

\section{Statistics}

We performed paired-samples $t$-test for normally distributed data and Wilcoxon signed-rank test for non-normally distributed data to compare the pretreatment and posttreatment numeric variables. McNemar's test was used to analyze categorical data. Spearman's rank correlation coefficients were used to evaluate the relationship between self-reported symptoms and affected arm volume. Spearman's rank correlation coefficients were accepted as follows: 0.81-1.0 as excellent, $0.61-0.80$ very good, $0.41-0.60$ good, $0.21-0.40$ fair, and $0-0.20$ poor. ${ }^{17}$ SPSS version 17 (SPSS, Inc., Chicago, IL) was used for all statistical analyses. $p$-Values less than 0.05 were considered to represent a significant difference.

\section{Results}

Demographic and disease characteristics of 25 women with subclinical lymphedema are presented in Table 2.

The mean time for the development of subclinical lymphedema after breast cancer surgery was $19.4 \pm 10.4$ months. The mean duration of symptoms of subclinical lymphedema was $1.2 \pm 0.8$ months. There was no family history according to limb swelling. Stemmer's sign was negative in all patients. Cellulitis symptoms such as redness and warmth were not detected in any patients.

Swelling was the most frequently reported upper extremity symptom in subclinical lymphedema patients $(88 \%)$. Seventy-two percent of the patients reported tightness, $60 \%$ reported heaviness, and $40 \%$ reported numbness before the treatment. All self-reported upper extremity symptoms declined significantly after the treatment (Table 3 ).

The mean volume of the affected arm decreased significantly at the end of the treatment $(p=0.01)$. The mean percentage volume difference between the affected and unaffected arm also decreased at the end of the treatment $(p<0.001)$. The mean Q-DASH and HADS-anxiety/depression scores decreased significantly after the treatment $(p<0.05)$. The mean SF-36 PCS and MCS scores increased significantly after the treatment $(p<0.05)$. The changes in affected arm volume, percentage volume difference, Q-DASH, HADS-anxiety/ depression, and SF-36 PCS and MCS scores before and after the treatment are shown in Table 4.

Table 1. Home-Based Exercise Program to Prevent Development of Lymphedema

Exercise Definition

1. Abdominal breathing exercises

2. Cervical range of motion exercises

3. Pectoral stretching exercises

4. Shoulder roll exercises

5. Shoulder stretching exercises

6. Biceps strengthening exercises

7. Forearm pronation/supination exercises

8. Wrist strengthening exercises

9. Hand open and close exercises
Sit down in an upright position. Put both hands on the abdomen. Take a deep breath through the nose, allow the abdomen to rise and hold for 5 seconds. Then breathe out through the mouth and allow the abdomen to flatten.

Sit down in an upright position. Bend the head forward as far as possible, letting it hang with the chin close to the chest. Extend the head back as far as possible, looking at the ceiling. Turn the head to the right as far as possible, looking over the right shoulder. Turn the head to the left as far as possible, looking over the left shoulder. Tilt the head to the right, bringing the right ear close to the shoulder as much as possible. Tilt the head to the left, bringing the left ear close to the shoulder as much as possible. Hold for at least 5 seconds for each exercise.

Sit down in an upright position. Squeeze the shoulder blades together and allow chest expansion.

Sit down in an upright position. Roll the shoulders forward and then backward.

Stand up straight. Hold a stick with the hands to push both arms forward and up as much as possible. Then push the affected arm to the side and up as much as possible. Then push both arms from side to side with elbow at $90^{\circ}$. Hold for at least 5 seconds for each exercise.

Sit down in an upright position. Hold a $0.5 \mathrm{~L}$ water bottle in the affected hand with the palm facing up. Place the affected arm in front of the body with elbow at $90^{\circ}$. Curl the weights up to the chest and lean back. Hold for at least 5 seconds.

Sit down in an upright position. Place the affected arm on the table with elbow straight. Turn the palm downward and upward. Hold for at least 5 seconds.

Sit down in an upright position. Hold a $0.5 \mathrm{~L}$ water bottle in the affected hand with the palm facing down. Raise the hand up and down. Then turn the palm upward and downward. Hold for at least 5 seconds for each exercise.

Sit down in an upright position. Hold the affected hand above the level of the heart with elbow at $90^{\circ}$. Open and close the fingers. Hold for at least 5 seconds. 
Table 2. Demographic and Disease Characteristics of The Patients With SubClinical Lymphedema (Mean \pm Standard Deviation or \%)

\begin{tabular}{|c|c|}
\hline & $\begin{array}{c}\text { Women } \\
\text { with subclinical } \\
\text { lymphedema }(\mathrm{n}=25)\end{array}$ \\
\hline Age (years) & $51.1 \pm 10.4$ \\
\hline Education (years) & $6.9 \pm 3.4$ \\
\hline \multicolumn{2}{|l|}{ Occupation $(\%)$} \\
\hline Housewife & 72 \\
\hline Worker & 20 \\
\hline Retired & 8 \\
\hline \multicolumn{2}{|l|}{ Comorbidity (\%) } \\
\hline No medical problem & 56 \\
\hline One or more medical problems & 44 \\
\hline BMI $\left(\mathrm{kg} / \mathrm{m}^{2}\right)$ & $23.5 \pm 4.5$ \\
\hline \multicolumn{2}{|l|}{ Breast cancer type $(\%)$} \\
\hline Infiltrative ductal carcinoma & 92 \\
\hline Infiltrative lobular carcinoma & 8 \\
\hline \multicolumn{2}{|l|}{ Breast cancer stage $(\%)$} \\
\hline Stage 1 & 28 \\
\hline Stage 2 & 56 \\
\hline Stage 3 & 16 \\
\hline \multicolumn{2}{|l|}{ Type of operation $(\%)$} \\
\hline MRM + ALND & 80 \\
\hline MRM + SLNB & 20 \\
\hline Lymph nodes removed $(n)$ & $15.7 \pm 8.7$ \\
\hline Positive lymph nodes $(n)$ & $1.8 \pm 0.5$ \\
\hline \multicolumn{2}{|l|}{ Adjuvant therapy $(\%)$} \\
\hline Radiotherapy & 52 \\
\hline Chemotherapy & 80 \\
\hline Endocrine therapy & 72 \\
\hline
\end{tabular}

ALND, axillary lymph node dissection; BMI, body mass index; MRM, modified radical mastectomy; SD, standard deviation; SLNB, sentinel lymph node biopsy.

The Spearman correlation analysis demonstrated that tightness and numbness were found to have a good correlation with affected arm volume ( $r=0.45$ and 0.37 , respectively, $p=0.02$ and 0.04 , respectively). There were no correlations between the other symptoms and affected arm volume $(r=0.07$ and $p=0.73$ for swelling; $r=0.11$ and $p=0.57$ for heaviness).

\section{Discussion}

Early diagnosis and adequate treatment of subclinical lymphedema are important to prevent development of clinically

Table 3. Change in Self-Reported UpPer EXTREMITY Symptoms of THE Patients WITH SUbCLINICAL LYMPHEDEMA After the Treatment

\begin{tabular}{lccc}
\hline & $\begin{array}{c}\text { Before treatment } \\
(\%)\end{array}$ & $\begin{array}{c}\text { After treatment } \\
(\%)\end{array}$ & $\mathrm{p}^{\mathrm{a}}$ \\
\hline Swelling & 88 & 24 & $<0.001$ \\
Tightness & 72 & 12 & $<0.001$ \\
Heaviness & 60 & 8 & $<0.001$ \\
Numbness & 40 & 4 & 0.008
\end{tabular}

\footnotetext{
${ }^{\mathrm{a}}$ Statistically significant difference.
}

Table 4. The Changes in Affected Arm Volume, Percentage Volume Difference, Quick Disabilities of Arm, Shoulder, and HANd, Short Form-36 Physical Component Summary and Mental Component Summary Scores, Hospital AnXiety AND Depression Scale ANXIETy AND Depression Scores of the Patients with Subclinical Lymphedema AfTer the Treatment

\begin{tabular}{lccc}
\hline & $\begin{array}{c}\text { Before } \\
\text { treatment }\end{array}$ & $\begin{array}{c}\text { After } \\
\text { treatment }\end{array}$ & $\mathrm{p}^{\mathrm{a}}$ \\
\hline $\begin{array}{c}\text { Affected arm } \\
\text { volume (mL) }\end{array}$ & $1392.0 \pm 478.9$ & $1330.1 \pm 376.9$ & 0.01 \\
$\begin{array}{c}\text { Percentage } \\
\text { volume }\end{array}$ & $7.2 \pm 3.3$ & $4.4 \pm 2.8$ & $<0.001$ \\
difference & & & \\
between both & & & \\
$\quad$ arms & & & \\
Q-DASH & $40.3 \pm 19.7$ & $28.3 \pm 15.5$ & $<0.001$ \\
SF-36 & & & \\
PCS & $60.1 \pm 17.9$ & $76.6 \pm 17.6$ & $<0.001$ \\
MCS & $70.1 \pm 18.5$ & $75.5 \pm 16.1$ & 0.01 \\
HADS & & & \\
Anxiety & $3.8 \pm 3.2$ & $2.8 \pm 2.3$ & 0.01 \\
Depression & $2.8 \pm 2.1$ & $2.1 \pm 1.9$ & 0.04 \\
\hline
\end{tabular}

${ }^{\text {a }}$ Statistically significant difference.

HADS, Hospital Anxiety and Depression Scale; MCS, mental component summary; PCS, physical component summary; QDASH, Quick-The Disabilities of the Arm, Shoulder, and Hand; SF36 , short form-36.

apparent lymphedema, allowing to maintain upper extremity functioning and quality of life because of its reversible property. In this phase, the accumulation of fluid is not clinically apparent, and thus, the diagnostic test ability to detect subclinical lymphedema is limited. ${ }^{7,8}$ On the contrary, there is no consensus on the management of subclinical lymphedema.

In several studies, optoelectronic perometry and bioimpedance spectroscopy have been used for detection of subclinical lymphedema. ${ }^{5,-10}$ However, these techniques are expensive and their applications are difficult in busy outpatient clinics. It has been suggested that patient self-reported symptoms such as limb swelling, heaviness, numbness, tightness are sensitive to the development of lymphedema in early stage. ${ }^{18,19}$ A combination of self-reported symptoms and limb volume measurements may be useful to detect subclinical lymphedema. The Dutch Institute for Healthcare Improvement recommended that if the volume difference between the limbs is $5 \%-10 \%$, it is suggested to start the treatment protocol for subclinical lymphedema. The symptoms such as swelling, tingling, and heaviness are also important for patients to recognize preclinical lymphedema. ${ }^{6}$ According to these recommendations, subclinical lymphedema was determined in patients who have volume difference between the limbs, less than $10 \%$, and at least one of the self-reported symptoms in this study.

Although lacking scientific evidence, the National Lymphedema Network informs breast cancer survivors about risk-reduction practice and exercises. Exercise is essential for both prevention and treatment of lymphedema and all subjects with breast cancer surgery should be encouraged to exercise. Exercise increases venous and lymphatic fluid return to the circulatory system and out of the swollen areas via 
improvement of muscle pump function. It also improves the range of movement and limb strength. The exercise program should include lymphedema remedial exercises, flexibility exercises, stretching exercises, strengthening exercises, and aerobic exercises. The exercise program should be performed slowly and progress gradually. Gentle and moderate physical activity and exercise did not increase the risk of lymphedema. Higher intensity exercise will promote inflammation and injury and should be avoided in patients at risk for lymphedema. ${ }^{16,20}$ Obesity is a major risk factor for developing secondary lymphedema in breast cancer patients. Interventions should include dietary advice and exercise programs to achieve reduction of weight in patients with body mass index $>25$. $^{6,21,22} \mathrm{We}$ recommended to patients a home-based treatment program that included skin care, information about lymphedema and its risk factors, and specific exercise protocol for 1 month. At the end of the program, we detected that volumetric values of the affected limb were significantly decreased. In the literature, studies about subclinical lymphedema used compression garments in addition to exercise, skin care, and information about lymphedema risk factors. ${ }^{5,9}$ Stout-Gergich et al. prescribed a $20-30 \mathrm{mmHg}$ compression garment for 4 weeks to patients with subclinical lymphedema and found a significant mean volume reduction $(48 \mathrm{~mL})$ after the intervention. ${ }^{5}$ Soran et al. reported that patients with subclinical lymphedema underwent short-term education, infection precautions, compression garment, education about exercise in the intervention group, and only clinical followups with circumferential arm measurements in the control group. Progression to clinical lymphedema occurred in $4.4 \%$ of the intervention group, whereas it was $36.4 \%$ in the control group. ${ }^{9}$ We did not recommend compression garment to the patients, because its usage is difficult. Nevertheless, mean volume reduction of $62 \mathrm{~mL}$ and volume difference reduction of $2.8 \%$ were found in the present study. Similar to Soran et al., progression to clinical lymphedema was detected in $4 \%$ of the patients (only one patient). The complex decongestive therapy was applied to this patient.

The Dutch Institute for Healthcare Improvement reported that characteristic clinical presentation is an essential step in the diagnosis of early lymphedema. ${ }^{6}$ In the present study, we found that the most frequent symptoms in patients with subclinical lymphedema were self-reported swelling and tightness. The frequencies of the self-reported symptoms were $88 \%$ for swelling, $72 \%$ for tightness, $60 \%$ for heaviness, and $40 \%$ for numbness. Similarly, Armer et al. reported that the symptoms experienced most commonly among patients with lymphedema were swelling (63\%), heaviness (60\%), and numbness $(38 \%) .{ }^{19} \mathrm{We}$ also found that tightness and numbness had a good correlation with affected arm volume. This result is in line with those reported by other studies. ${ }^{23,24}$ Ridner et al. detected that self-reported tightness significantly correlated with circumferential measurements and bioimpedance measurements in healthy volunteers and patients with lymphedema. ${ }^{23}$ Bundred et al. reported a strong correlation between volume measurements using bioimpedance analysis and self-reported symptoms. ${ }^{24}$

We detected significant improvement in all self-reported symptoms at the end of treatment. This study is the first, to our knowledge, to evaluate the effects of treatment on selfreported symptoms of subclinical lymphedema. The previous studies evaluated the effects of treatment on symptoms in clinical lymphedema. ${ }^{25,26}$ Finlay et al. found that selfreported swelling and heaviness reduced in the objectively measured limb volume in patients with clinical lymphedema. ${ }^{25}$ Cormier et al. reported that subjective symptoms generally become worse with increasing limb volume in breast cancer survivors. ${ }^{26}$ These findings were consistent with data from this study. Our results suggest that the experience of these symptoms is linked to limb volume; therefore, treatments targeting a reduction in limb volume should be effective in reducing the incidence.

Lymphedema causes pain and discomfort and it reduces upper extremity function and quality of life in patients with breast cancer. It is also associated with psychosocial problems. ${ }^{4}$ When lymphedema is detected in earlier stages, therapeutic management is more likely to be effective in improving outcomes and quality of life. ${ }^{1}$ In the present study, arm functions, quality of life, and anxiety and depression symptoms of patients with subclinical lymphedema significantly improved after treatment. To our knowledge, there is no study about functioning, quality of life, and psychiatric morbidity in subclinical lymphedema patients.

There were some limitations in our study. First, the sample size of the study was limited. A larger sample size would have been more powerful. Second, there was no control group in the study. We did not assess subclinical lymphedema patients without treatment.

In conclusion, it is crucial to have early detection and treatment of subclinical lymphedema to prevent the development of established lymphedema in patients with breast cancer. Results from this study demonstrate that information about lymphedema and its risk factors, skin care, and a homebased specific exercise program improves self-reported symptoms, volume measurements, upper extremity functions, quality of life, and psychiatric morbidities. Future randomized controlled studies with a larger sample size are needed to confirm the effects of early detection and treatment of subclinical lymphedema in breast cancer survivors.

\section{Author Disclosure Statement}

No competing financial interests exist.

\section{References}

1. Chance-Hetzler J, Armer J, Van Loo M, et al. Prospective lymphedema surveillance in a clinic setting. J Pers Med 2015; 5:311-325.

2. Schmitz KH. Balancing lymphedema risk: exercise versus deconditioning for breast cancer survivors. Exerc Sport Sci Rev 2010; 38:17-24.

3. Kamalı Polat A, Ufuk Karabacak, Mutlu V, Tomak L, Bilgici A. early diagnosis of lymphedema after breast cancer treatment: Bio-impedance spectroscopy. J Breast Health 2017; 13:83-87.

4. Cemal Y. Preventative measures for lymphedema: Separating fact from fiction. Am Coll Surg 2011; 213:543-551.

5. Stout-Gergich NL, Pfalzer LA, McGarvey C, Springer B, Gerber LH, Soballe P. Preoperative assessment enables the early diagnosis and successful treatment of lymphedema. Cancer 2008; 112:2809-2819.

6. Damstra R, Halk AB; Dutch Working Group on Lymphedema. The Dutch lymphedema guidelines based on the International Classification of Functioning, Disability, and 
Health and the chronic care model. J Vasc Surg 2017; 5: 756-765.

7. Levenhagen K, Davies C, Perdomo M, Ryans K, Gilchrist L. Diagnosis of upper-quadrant lymphedema secondary to cancer: Clinical practice guideline from the Oncology Section of APTA. Rehab Oncol 2017; 35:E1-E18.

8. Shah C, Arthur DW, Wazer D, Khan A, Ridner S, Vicini F. The impact of early detection and intervention of breast cancer-related lymphedema: A systematic review. Cancer Med 2016; 5:1154-1162.

9. Soran A, Ozmen T, McGuire KP, et al. The importance of detection of subclinical lymphedema for the prevention of breast cancer-related clinical lymphedema after axillary lymph node dissection; a prospective observational study. Lymphat Res Biol 2014; 12:289-294.

10. Johansson K, Branje E. Arm lymphoedema in a cohort of breast cancer survivors 10 years after diagnosis. Acta Oncol 2010; 49:166-173.

11. Karges JR, Mark BE, Stikeleather SJ, Worrell TW. Concurrent validity of upper extremity volume estimates: Comparison of calculated volume derived from girth measurements and water displacement volume. Phys Ther 2003; 83:134-145.

12. Düger T, Yakut E, Öksüz Ç, et al. Anketi Türkçe uyarlamasının güvenirliği ve geçerliği [Disabilities of the arm, sholder and hand-DASH]. Fizyoter ReHabil 2006; 17:99-107.

13. Singer S, Kuhnt S, Götze $\mathrm{H}$, et al. Hospital anxiety and depression scale cutoff scores for cancer patients in acute care. Br J Cancer 2009; 100:908-912.

14. Aydemir O, Guvenir T, Kuey L, Kültür S. The reliability and validity of Turkish form of Hospital Anxiety and Depression Scale. Turk Psikiyatri Derg 1997; 8:280-287.

15. Pinar R. Reliability and construct validity of the SF-36 in Turkish cancer patients: Qual Life Res 2005; 14:259-264.

16. National Lymphedema Network Medical Advisory Committee. Topic: Lymphedema risk reduction practices. Journal. Internet. cited 2008 (September 17). Available from: www.lymphnet.org/pdfDocs/nlnriskreduction.pdf Accessed September 15, 2018.

17. Feise RJ, Menke JM. Functional Rating Index: A new valid and reliable instrument to measure the magnitude of clinical change in spinal conditions. Spine 2001; 26: 78-87.

18. Norman SA, Localio AR, Potashnik SL, et al. Lymphedema in breast cancer survivors: incidence, degree, time course, treatment, and symptoms. J Clin Oncol 2008; 27: 390-397.

19. Armer J, Radina M, Porock D, Culbertson SD. Predicting breast cancer-related lymphedema using selfreported symptoms. Nurs Res 2003; 52:370-379.

20. Kwan ML, Cohn JC, Armer JM, Stewart BR, Cormier JN. Exercise in patients with lymphedema: A systematic review of the contemporary literature. J Cancer Surviv 2011; 5: 320-336.

21. Erdogan Iyigun Z, Duymaz T, Ilgun AS, et al. Preoperative lymphedema-related risk factors in early-stage breast cancer. Lymphat Res Biol 2018; 16:28-35.

22. Yoon JA, Shin YB, Shin MJ, et al. An assessment of the relationship between abdominal obesity and the severity of upper extremity lymphedema. Lymphat Res Biol 2018; 16: 458-463.

23. Ridner SH, Stewart BR, Armer JM. Comparison of upper limb volume measurement techniques and arm symptoms between healthy volunteers and individuals with known lymphedema. Lymphology 2007; 40:35-46.

24. Bundred NJ, Stockton C, Keeley V, et al; The Investigators of BEA/PLACE Studies. Comparison of multifrequency bioimpedance with perometry for the early detection and intervention of lymphoedema after axillary node clearance for breast cancer. Breast Cancer Res Treat 2015; 151:121-129.

25. Finlay B, Ullah S, Piller N. Relationship between pain, tightness, heaviness, perceived limb size, and objective limb size measurements in patients with chronic upper-limb lymphoedema. J Lymphoedema 2013; 8:10-16.

26. Cormier JN, Xing Y, Zaniletti I, Askew RL, Stewart BR, Armer JM. Minimal limb volume change has a significant impact on breast cancer survivors. Lymphology 2009; 42: 161-167.

Address correspondence to: Asli Gençay Can, MD University of Health Science

Diskapi Yildirim Beyazit Research and Training Hospital Department of Physical Medicine and Rehabilitation

Altında $\breve{g}$

Ankara 06800

Turkey

E-mail: asligencay@yahoo.com 